\title{
Time course of the development of non-alcoholic hepatic steatosis in response to high-fat diet-induced obesity in rats
}

\author{
Marie-Soleil Gauthier ${ }^{1}$, Roland Favier ${ }^{2}$ and Jean-Marc Lavoie ${ }^{1} *$ \\ ${ }^{1}$ Département de kinésiologie, Université de Montréal, C.P. 6128, Succ. centre-ville, Montréal, Québec, Canada H3C 3J7 \\ ${ }^{2}$ Équipe Mixte INSERM 221, Laboratoire de Bioénergétique Fondamentale et Appliquée, Université Joseph Fourier, Grenoble, \\ France
}

(Received 2 June 2005 - Revised 31 August 2005 - Accepted 28 September 2005)

\begin{abstract}
The aim of the study was to characterize the time course of the development of high-fat diet-induced hepatic steatosis and its relation to body fat accretion and changes in plasma lipid profile. Female Sprague-Dawley rats were high-fat fed (HF; 42\%, kJ) for 1, 2, 4, 6, 12 and 16 weeks and compared to standard fed rats (SD). Data obtained from HF rats were further analysed by classifying the animals into obesity-prone and obesityresistant. In HF rats, liver lipid content increased rapidly by approximately $200 \%$ during the first 2 weeks, decreased almost to baseline levels between weeks 2 and 6, and re-increased by $17 \%$ between weeks 6 and $16(P<0 \cdot 05)$. Body weight, body fat accretion, plasma leptin, NEFA and glycerol concentrations were higher in HF than in SD rats $(P<0.05)$. These higher values were established in 2 weeks and the differences between the groups did not further enlarge from weeks 2 to 16. Obesity-prone rats depicted higher body weight and body fat accretion than obesity-resistant and SD rats. Surprisingly, however, liver lipid content was the same in obesity-prone as in obesity-resistant rats as they were both higher than in SD rats (weeks 2 and 16; $P<0.05$ ). Our data support the hypothesis that the liver acts as a systemic buffer, largely increasing its lipid content in the early stage of high-fat feeding. Our results also suggest that the development of non-alcoholic hepatic steatosis is more linked to dietary fat ingestion than to body weight gain.
\end{abstract}

Fatty liver: Intra-abdominal fat: Plasma lipid profile: NAFLD: Obesity resistant

It is well established that the ingestion of a high-fat diet promotes obesity and the development of the metabolic syndrome in both rodents and man (Hill et al. 1992; Ghibaudi et al. 2002; Satia-Abouta et al. 2002; Axen et al. 2003). Besides increased fat accretion in adipocytes and hyperlipidaemia, recent data also indicate that such high-fat diets result in non-alcoholic hepatic steatosis, which is characterized by an excessive accumulation of triacylglycerol (TAG) within hepatocytes. Non-alcoholic hepatic steatosis is now recognized as an integral part of the metabolic syndrome and has been associated with the development of insulin resistance and insulin signalling defects (Marceau et al. 1999; Kim et al. 2000; Reue et al. 2000; Saltiel \& Kahn, 2001; Seppala-Lindroos et al. 2002; Samuel et al. 2004). Furthermore, an accumulating body of evidence suggests that non-alcoholic hepatic steatosis may progress to advanced liver disease in a proportion of patients (Day, 2002).

Recent works conducted in our laboratory, using the highfat diet regimen approach, have led us to postulate that, opposite to what is generally assumed, lipids do not accumulate in a linear fashion in the liver (Gauthier et al. 2003, 2004). In these works (Gauthier et al. 2003, 2004), we hypothesized that hepatic steatosis develops rapidly when rats undertake a high-fat diet regimen but does not increase linearly over time and might even decrease if high-fat feeding is pursued for a longer period of time. This observation would be consistent with the suggestion that the liver acts as a systemic lipid buffer during periods of high lipid influx (Frayn, 2002; Gauthier et al. 2003) and may be the first organ to store excessive amounts of fatty acids (Westerbacka et al. 2005). One way to better circumscribe the role of the liver as a 'fat trapper' in response to high-fat feeding is to establish the time course of the development of hepatic steatosis. Surprisingly, very little information on the time course of the development of high-fat diet-induced obesity and hepatic steatosis is available. Such information may be useful not only to better characterize liver lipid infiltration but to better understand how a lipid load is managed by the body over time. Since hepatic steatosis often develops in conjunction with lipid accumulation in adipocytes and changes in blood lipid profile, these parameters must be considered to better understand how hepatic steatosis develops. Thus, the main goal of the present study was to gain some information on the development of hepatic steatosis by characterizing the time sequence (1-16 weeks) of high-fat diet-induced liver lipid infiltration in rats.

Sprague-Dawley rats show large inter-individual differences in susceptibility to weight gain when submitted to high-fat feeding (Lauterio et al. 1994; Levin et al. 1997; Wang et al. 1998; Ghibaudi et al. 2002). High-fat fed (HF) obesity-resistant rats seem to be protected against weight

Abbreviations: HF, high-fat fed; HF-OP, high-fat fed obesity-prone; HF-OR, high-fat fed obesity-resistant; SD, standard fed; TAG, triacylglycerol.

* Corresponding author: Dr Jean-Marc Lavoie, fax +1 514343 2181, email jean-marc.lavoie@umontreal.ca 
gain and adipocyte fat accretion (Pagliassotti et al. 1993, 1994, 1995; Lauterio et al. 1994; Levin et al. 1997; Wang et al. 1998; Commerford et al. 2000; Ghibaudi et al. 2002) but not consistently from high-fat diet-induced increase in plasma NEFA (Commerford et al. 2000; Ghibaudi et al. 2002; Ji et al. 2005). Liver lipid infiltration is highly related to the amount of circulating NEFA (Sniderman et al. 1998). It is, however, unknown if obesity-resistant rats are protected against high-fat diet-induced hepatic steatosis. Thus, to further characterize liver lipid infiltration in response to high-fat feeding, we proceeded to a second analysis of the results where HF rats that had the highest and the lowest weight gain were designated obesity-prone (HF-OP) and obesity-resistant (HF$\mathrm{OR})$, respectively, and compared to control standard fed (SD) rats.

\section{Methods}

\section{Animal care}

Female Sprague-Dawley strain rats (n 119; Charles River, St Constant, PQ, Canada), weighing 180-200 g (6 weeks of age) upon their arrival were housed individually and had ad libitum access to food and tap water. Their environment was controlled in terms of light $(12 \mathrm{~h}$ light-dark cycle starting at 06.00 hours), humidity and room temperature $\left(20-23^{\circ} \mathrm{C}\right)$. All experiments described in this report were conducted according to the directives of the Canadian Council on Animal Care.

\section{Diet protocol}

Animals were randomly assigned to either the SD or HF diet $3 \mathrm{~d}$ after their arrival. The HF diet consisted of $42 \%$ lipid, $36 \%$ carbohydrate and $22 \%$ protein $(\mathrm{kJ})$ and was provided in small pellets from ICN Pharmaceuticals (New York, USA). The lipids included in the high-fat diet consisted of $80 \%$ lard (41\% saturated fat, 44\% MUFA and 9\% PUFA; $\mathrm{g}$ fatty acids/100 $\mathrm{g}$ of lard), and $20 \%$ corn oil (13\% saturated fat, $25 \%$ MUFA and $58 \%$ PUFA; g fatty acids/100 $\mathrm{g}$ of corn oil). The standard diet ( $12.5 \%$ lipid, $63.2 \%$ carbohydrate and $24.3 \%$ protein; kJ) consisted of usual pellet rat chow (Agribrands Purina Canada, Woodstock, Ontario, Canada). Details of the diets have been presented elsewhere (Gauthier et al. 2003). Twelve rats were immediately killed after their $3 \mathrm{~d}$ acclimatization period to provide baseline values for the dependent variables. Rats on the high-fat diet were killed after $1,2,4,6,8,12$ and 16 weeks (n 9-10), while control rats on the SD diet were killed only after 2, 6, 12 and 16 weeks $(n 9-10)$. Body weight and food intake were monitored daily in all rats. For the second part of the study, HF rats were classified according to their weight gain during their respective period of high-fat feeding (2, 6, 12 and 16 weeks). The five rats showing the highest weight gain and the five showing the lowest weight gain were further classified as HF-OP and HF-OR.

\section{Blood and tissue sampling}

At the end of their respective dietary manipulation, all animals were killed between 09.00 and 12.00 hours. Food was removed from the animals' cage $2-3 \mathrm{~h}$ before being killed. After complete anaesthesia (sodium pentobarbital, $50 \mathrm{mg} / \mathrm{kg}$, intraperitoneal injection), the abdominal cavity was rapidly opened following the median line of the abdomen. Blood was rapidly $(<45 \mathrm{~s})$ and simultaneously drawn from the abdominal vena cava (about $4 \mathrm{ml}$ ) and the hepatic portal vein (about $1.5 \mathrm{ml}$ ) into syringes pretreated with EDTA $(15 \%)$. Blood was centrifuged $\left(3000 \mathrm{rpm}\right.$ for $8 \mathrm{~min}, 4^{\circ} \mathrm{C}$ ) and the plasma kept for further analyses. Liver, muscles and fat deposits were excised and weighed in the following order: liver, mesenteric fat, urogenital fat, retroperitoneal fat and subcutaneous fat. All tissue samples were frozen in liquid nitrogen immediately after being weighed. The liver median lobe was freeze-clamped and used for glycogen and TAG determinations. Mesenteric fat pad consisted of adipose tissue surrounding the gastro-intestinal tract from the gastro-oesophageal sphincter to the end of the rectum with special care taken in distinguishing and removing pancreatic cells. Urogenital fat pad included adipose tissue surrounding the kidneys, ureters and bladder as well as ovaries, oviducts and uterus. Retroperitoneal fat pad was taken as that distinct deposit behind each kidney along the lumbar muscles. For subcutaneous deposit measurement, a rectangular piece of skin was taken on the right side of each animal, from the median line of the abdomen to the spine and the right hip to the first rib as described by Krotkiewski \& Bjorntorp (1976). All plasma and tissue samples were stored at $-78^{\circ} \mathrm{C}$ until analyses.

\section{Analytical procedures}

Plasma glucose concentration was determined with the use of a glucose analyser (Yellow Springs Instruments 2300, Yellow Springs, OH, USA). Insulin concentrations were measured with commercially available RIA kits (Medicorp, Montréal, PQ, Canada; ICN Pharmaceuticals). Leptin concentrations were measured with commercially available RIA kits (Linco Research Inc., St Charles, MO, USA). Plasma NEFA levels were measured with a commercially available kit from Roche Diagnostics (Mannheim, Germany) and glycerol, TAG and $\beta$-hydroxybutyrate were measured with kits from Sigma Diagnostics (St Louis, MO, USA).

Liver TAG content was estimated from glycerol released after ethanolic $\mathrm{KOH}$ hydrolysis using a commercial kit (Sigma Diagnostics). Although this method does not discriminate between glycerol from phospholipids or TAG, Frayn \& Maycock (1980) have shown that omitting removal of phospholipids leads to only a $\pm 2 \%$ error in the determination of tissue TAG. Liver glycogen content was determined by use of the phenol-sulphuric acid reaction (Lo et al. 1970).

\section{Statistical analyses}

Values are expressed as means and standard errors. Statistical analyses were performed using a two-way ANOVA for nonrepeated measures design with diet and time as main effects at common time-points 2, 6, 12 and 16 weeks, excluding time-point 0 (since no diet treatment was given at that time). In a second step, the effect of time was analysed separately in $\mathrm{HF}$ and $\mathrm{SD}$ rats using a one-way ANOVA for non-repeated measures over time-points $0,1,2,4,6,8,12$ and 16 weeks in $\mathrm{HF}$ rats and time-points $0,2,6,12$ and 
16 weeks for SD rats. This second statistical analysis was performed to better characterize the effect of time in each diet including comparison with time 0 . In the second part of the study, HF-OP, HF-OR and SD rats were compared using a two-way ANOVA for non-repeated measures design with phenotype and time as main effects at time-points 2, 6, 12 and 16 weeks. Fisher's post-hoc test was used in the event of a significant $(P<0 \cdot 05) F$ ratio.

\section{Results}

Mean daily energy intake was significantly higher $(P<0.05)$ in rats that were fed the high-fat diet only for the first 2 weeks compared to SD counterparts (Fig. 1(A)). Fig. 1(B) illustrates the mean daily energy intake from week 1 to 16 only in rats that were fed either the high-fat or the standard diet for 16 weeks. This Fig. shows that on a high-fat diet, rats ingested more energy during the first few weeks and then lowered their daily energy intake to levels similar to SD rats. Rats on the high-fat diet depicted overall (from week 2 to 16) higher $(P<0.05)$ body weight compared to $\mathrm{SD}$ rats (Fig. 1(C)). Liver TAG content was higher $(P<0 \cdot 05)$ in $\mathrm{HF}$ than in SD rats at weeks 2 (about 200\%) and 16 (about $50 \%$ ) (Fig. 1(D)). In HF rats, liver TAG content increased by about 2-fold during the first 2 weeks of the diet $(P<0.05)$, to decrease during weeks 2 to $6(P<0.05)$ to a level that was not significantly $(P>0.05)$ different from baseline values anymore. Liver lipid infiltration re-increased when high-fat feeding was pursued over 6 weeks, HF rats showing liver TAG values significantly $(P<0 \cdot 05)$ higher than resting levels at weeks 8 and 16. There was no effect of time on liver TAG concentration in SD rats $(P>0 \cdot 05)$. Liver glycogen content was significantly $(P<0.05)$ lower in HF than in SD rats (Fig. 1(E)). There was no significant $(P>0.05)$ effect of time on liver glycogen levels.

\section{Fat depots}

The relative weights of the mesenteric, retroperitoneal and urogenital fat depots as well as plasma leptin concentrations were significantly $(P<0 \cdot 05)$ higher in $\mathrm{HF}$ than in SD rats throughout weeks 2-16 (Fig. 2). There was no statistical interaction in any of these responses indicating that high-fat feeding induced a rapid increase in these variables during the first 2 weeks and that the difference between HF and SD rats was not accentuated or diminished throughout the remaining weeks. The intra-abdominal fat depots and plasma leptin concentrations were significantly $(P<0.05)$ higher with time in both dietary conditions. The response of the subcutaneous fat pad was, however, somewhat different than the intraabdominal fat depots (Fig. 2(D)). Although overall higher $(P<0.05)$ values were found in HF compared to SD rats, the accumulation of subcutaneous lipids was rapidly increased in $\mathrm{HF}$ rats (first 2 weeks) to decrease during weeks $2-4$ and increase again until the end of the experiment.

\section{Plasma variables}

As for fat pads weight, plasma NEFA and glycerol concentrations were higher $(P<0.05)$ in HF than in SD rats throughout weeks 2-16, the difference between the two dietary groups being established during the first 2 weeks on the diets and remaining constant during the remainder of the study (Fig. 3(A, B)). Plasma NEFA and glycerol levels increased $(P<0.05)$ rapidly with time (first week) in HF rats while they were significantly $(P<0.05)$ increased only after 12 weeks when rats were fed the standard diet. No significant differences $(P>0.05)$ were found in plasma TAG between HF and $\mathrm{SD}$ rats $(P>0.05)$, the values increasing $(P<0.05)$ similarly on and after the sixth week in both dietary conditions (Fig. 3(C)). No significant $(P>0.05)$ differences were found in plasma $\beta$-hydroxybutyrate between the two dietary groups (Fig. 3(D)). Plasma $\beta$-hydroxybutyrate concentrations were found to be lower with time on and after the 12th week in $\mathrm{SD}$ rats only.

High-fat feeding was associated with small but significantly $(P<0.05)$ higher plasma glucose values (Fig. 4(A)) along with higher $(P<0.05)$ overall plasma insulin values in portal but not in peripheral blood (Fig. 4(B, C)).

\section{Comparisons of high-fat fed obesity-prone and obesity- resistant rats with control standard-fed rats}

To further characterize liver lipid infiltration in response to high-fat feeding, we proceeded to a second analysis of the results where $\mathrm{HF}$ rats that had the highest and the lowest weight gain were designated HF-OP and HF-OR, respectively, and compared to control SD rats. As expected, rats prone to high-fat diet-induced obesity (HF-OP) gained more weight than rats resistant to high-fat diet-induced obesity (HF-OR) and $\mathrm{SD}$ rats $(P<0.05$; Fig. 5(A)). Weight gain in HF-OR rats was similar $(P>0.05)$ to rats fed the standard diet with the exception of week 2 where they gained slightly more weight than the SD rats $(P<0 \cdot 05)$. Mean daily energy intake was significantly higher $(P<0.05)$ in $\mathrm{HF}-\mathrm{OP}$ rats than HFOR and SD rats except at week 12 (Fig. 5(B)). No difference in energy intake was found between HF-OR and SD rats with the exception of week 16 where HF-OR rats had lower energy intake than SD rats $(P<0 \cdot 05)$. Intra-abdominal and subcutaneous fat pad weights were heavier in $\mathrm{HF}-\mathrm{OP}$ rats than in the other two groups at each time-point $(P<0 \cdot 05$; Fig. $5(\mathrm{C}$, D)). HF-OR rats also showed heavier intra-abdominal fat pad weights than SD rats $(P<0 \cdot 05)$. Unexpectedly, no difference was found in liver TAG content between HF-OP and HFOR $(P>0.05)$, these two groups depicting higher liver TAG content than SD rats at weeks 2 and $16(P<0 \cdot 05$; Fig. 5(E)). Similarly to liver TAG, there were no significant differences $(P>0.05)$ in plasma NEFA levels between HF-OP and HFOR rats, both of these groups showing higher plasma NEFA levels than SD rats $(P<0.05$; Fig. $5(\mathrm{~F}))$.

\section{Discussion}

Although the significance of hepatic steatosis in relation to obesity and the metabolic syndrome has gained interest in recent years, there is a paucity of information on the development of this metabolic phenomenon. One of the advantages of using an animal instead of a human model to study obesity is that it is ethically easier to use a nutritional stimulus to examine how obesity develops instead of using obese versus nonobese comparisons. This approach was used in the present 


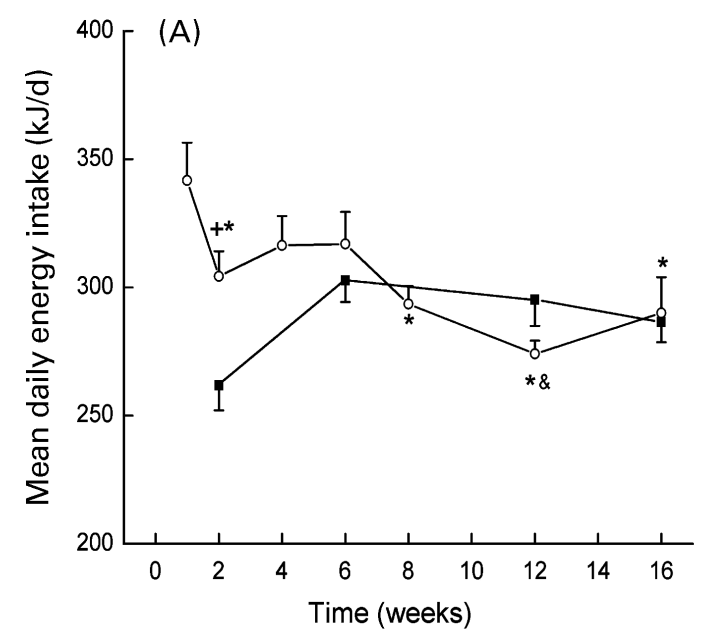

(B)
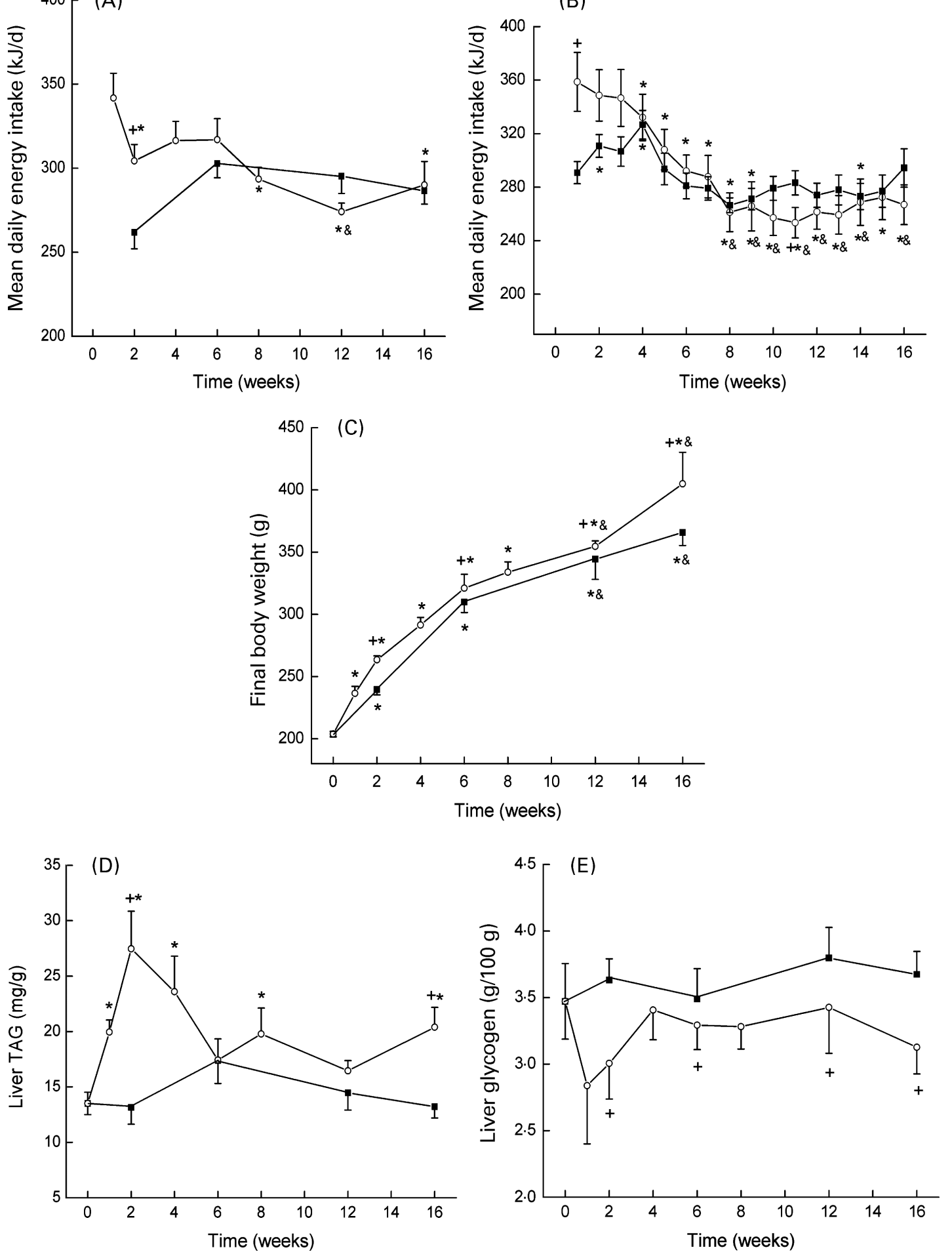

Fig. 1. Mean daily energy intake in rats killed at different times and calculated for the whole period of feeding (A), mean daily energy intake from weeks 1 to 16 only in rats that were killed after 16 weeks and calculated weekly (B), final body weight (C), liver triacylglycerol (TAG) concentration (D) and liver glycogen concentration $(E)$ in rats fed a standard $(\mathbf{\square}, n 9-10)$ or a high-fat $(O, n 9-10)$ diet. For details of procedures, see p. 274. Values are means with their standard errors depicted by vertical bars. ${ }^{+}$Mean value significantly different from standard fed rats $(P<0.05)$. ${ }^{*}$ Mean value significantly different from week 0 (significantly different from week 1 in $(A)$ and $(B)$ only) $(P<0.05)$. ${ }^{~}$ Mean value significantly different from week $6(P<0.05)$.

study to examine how high-fat diet-induced hepatic steatosis develops over time and in relation to other metabolic deteriorations. The present time-course data show that in response to an increased ingestion of fat, the liver becomes rapidly (1-2 weeks) and highly (about 2-fold) infiltrated with lipids, to decrease its lipid content in subsequent weeks (weeks 4-6), and becomes slowly re-infiltrated if the high-fat diet is pursued for a longer period of time (6-16 weeks). These observations 

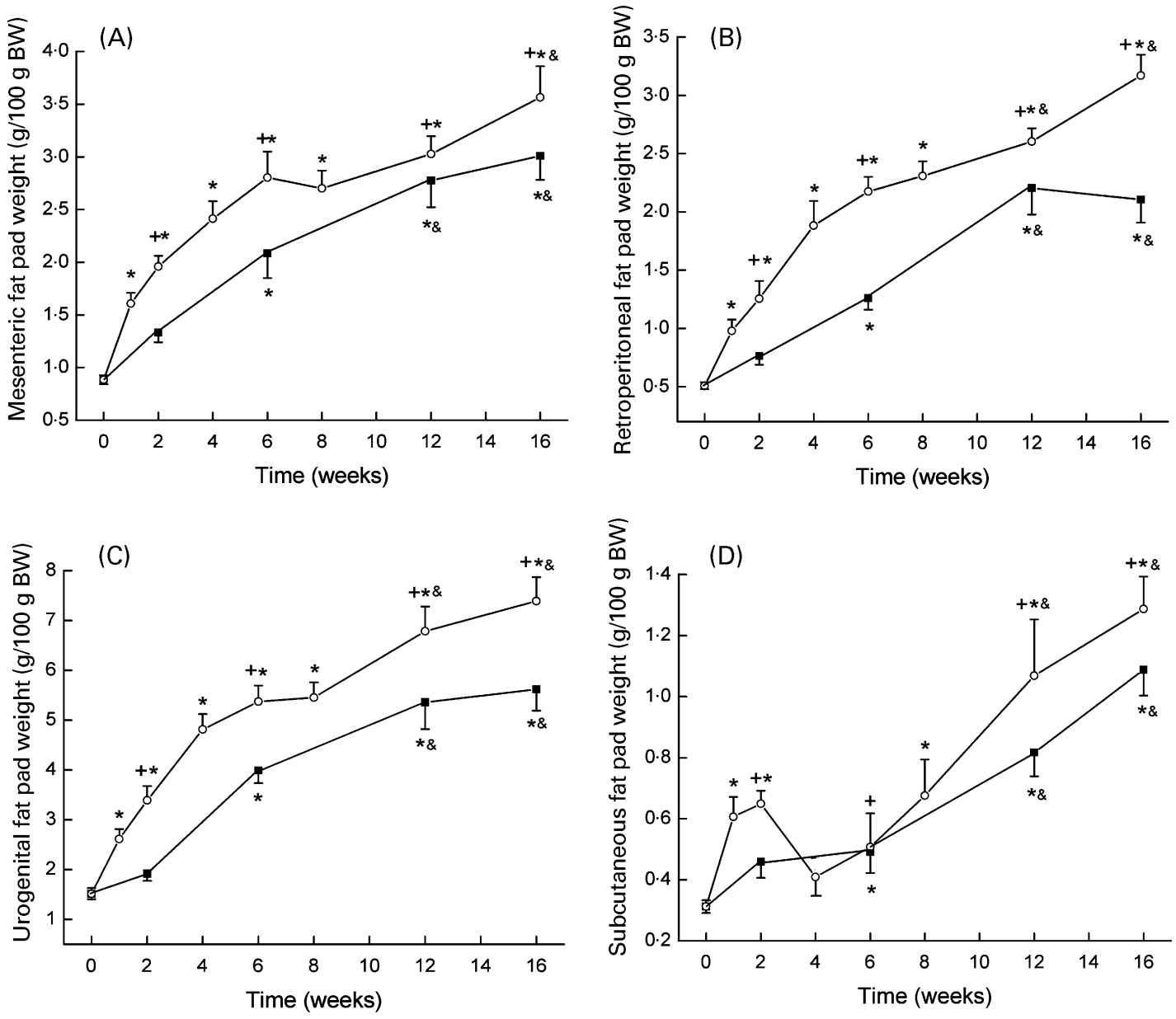

(E)

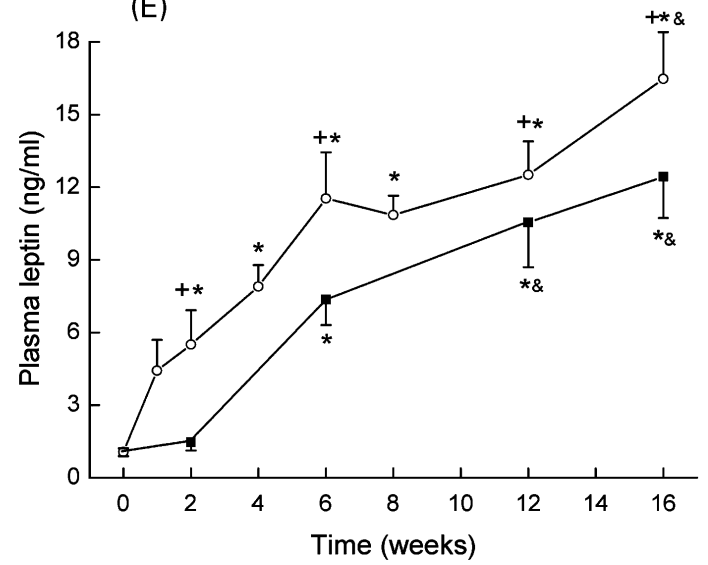

Fig. 2. Mesenteric (A), retroperitoneal (B), urogenital (C) and subcutaneous (D) fat pad weight relative to $100 \mathrm{~g}$ body weight (BW), and plasma leptin concentration $(E)$ in rats fed a standard $(\square, n 9-10)$ or a high-fat $(O, n 9-10)$ diet. For details of procedures, see p. 274 . Values are means with their standard errors depicted by vertical bars. ${ }^{+}$Mean value significantly different from standard fed rats $(P<0.05)$. ${ }^{*}$ Mean value significantly different from week $0(P<0.05)$. ${ }^{\circledR}$ Mean value significantly different from week $6(P<0.05)$.

suggest that high-fat feeding-induced hepatic steatosis is, at first, a transitory phenomenon that probably helps avoid the excessive accumulation of circulating lipids upon a sudden increase in fat ingestion. Previous studies have shown that hepatic steatosis can occur as early as after $3 \mathrm{~d}$ of high-fat feeding (Kraegen et al. 1991; Samuel et al. 2004). To our knowledge, the present data show for the first time that the time course of high-fat feeding-induced liver lipid infiltration occurs as a rapid and large increase in liver fat content during the first few weeks which can subsequently decrease, even if the high-fat diet is maintained, and slowly re-increase over time. However, the present data do not preclude that highfat feeding-induced hepatic steatosis cannot become a more stable phenomenon, as suggested by the week 16 values, if 

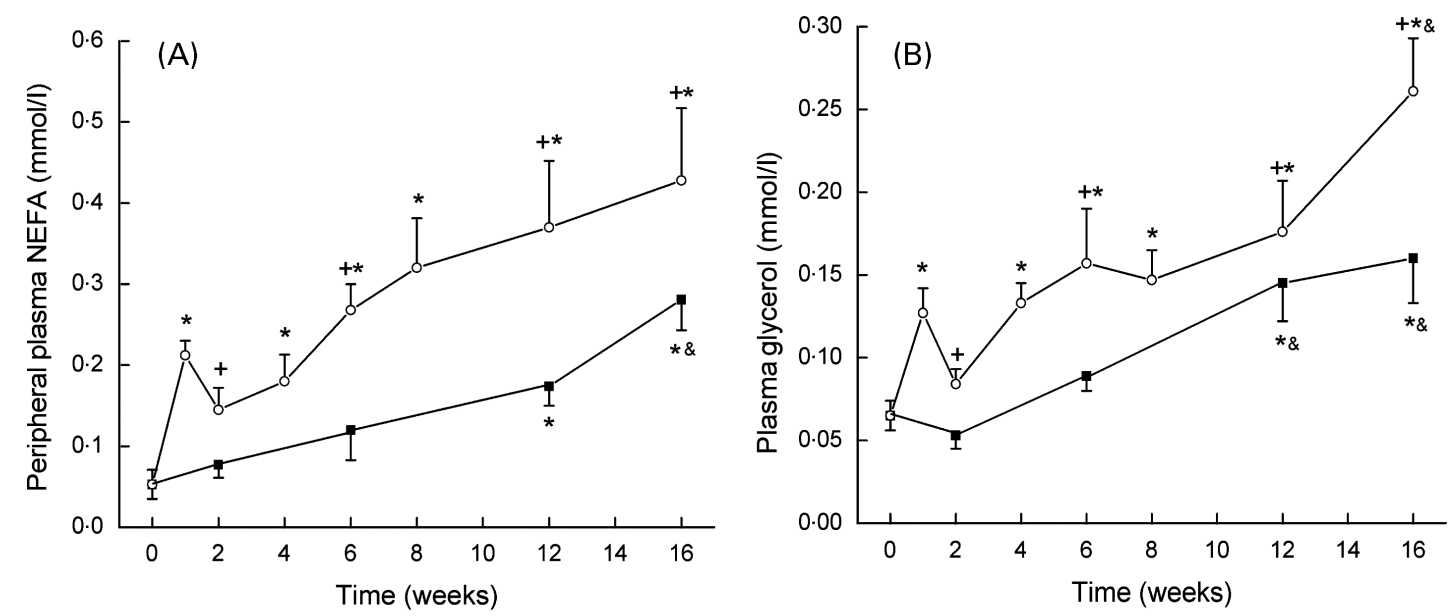

(C)
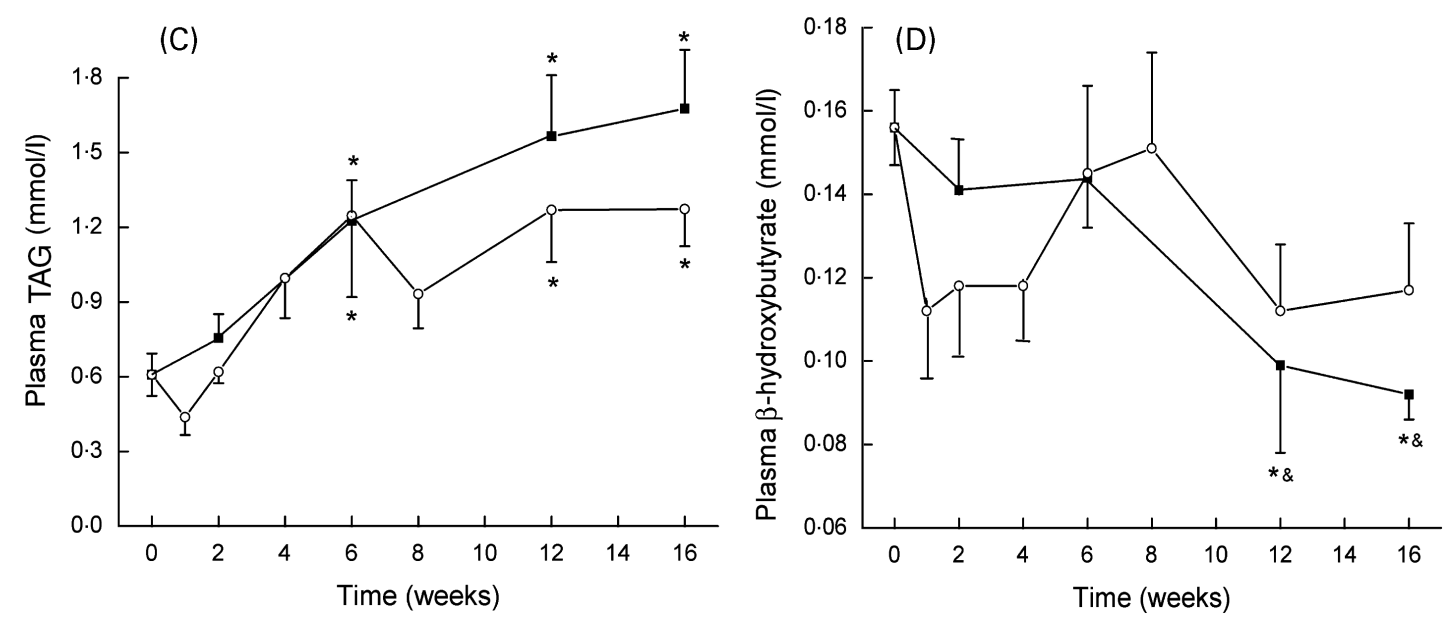

Fig. 3. Plasma NEFA (A), glycerol (B), triacylglycerol (TAG), (C) and $\beta$-hydroxybutyrate concentrations (D) in rats fed a standard ( $\square, n 9-10)$ or a high-fat $(\bigcirc, n$ 9-10) diet. For details of procedures, see p. 274. Values are means with their standard errors depicted by vertical bars. ${ }^{+}$Mean value significantly different from standard fed rats $(P<0.05)$. ${ }^{*}$ Mean value significantly different from week $0(P<0.05)$. ${ }^{\&}$ Mean value significantly different from week $6(P<0.05)$.

the diet or the obesity stimulus is pursued for a longer period of time. High-fat feeding for 10 months in male Wistar rats resulted in a large increase in liver TAG concentrations (Chalkley et al. 2002). It is also revealing to observe that in rats fed a standard diet, liver lipid content did not increase over time. This is in contrast to what is seen in the plasma and the adipocytes of these SD rats where lipids accumulate over time even if the lipid content of the diet remains unchanged. This suggests that liver lipid infiltration is more closely related to the diet than to ageing. The present findings on the development of hepatic steatosis are, however, limited to the high-fat diet model since the mechanisms leading to hepatic steatosis might be different with other types of diets (i.e. high sucrose) or in genetic models of obesity (i.e. $o b / o b$ mice and Zucker $\mathrm{fa} / \mathrm{fa}$ rats).

As expected, HF-OP rats showed higher intra-abdominal and subcutaneous fat than SD rats while HF-OR rats seemed largely protected against these high-fat diet-induced deleterious effects. Surprisingly however, at time when significant hepatic steatosis was present in HF rats (weeks 2 and 16), HF-OR rats depicted the same degree of liver lipid infiltration as HF-OP rats (Fig. 5(E)). In addition, HF-OR rats showed NEFA concentrations similar to HF-OP rats (Fig. 5(F)).
These results suggest that HF-OR rats might not be protected against high-fat feeding-induced liver lipid infiltration. This is in agreement with the recent work of Westerbacka et al. (2005), who found a close relationship between the amount of dietary fat and liver lipid content in man independent of body fat. These results are also in line with the suggestion that hepatic steatosis is independent of body weight gain and more closely related to circulating NEFA levels (Seppala-Lindroos et al. 2002). Overall, data from the present study indicate that, opposite to adipocyte fat accretion, highfat diet-induced hepatic steatosis does not develop linearly over time. Moreover, the HF-OP and HF-OR comparison reveals that high-fat feeding-induced hepatic steatosis is independent of the ability of the animal to resist body fat gain.

In agreement with the suggestion of Westerbacka et al. (2005), our data on high-fat diet-induced liver lipid infiltration appear to indicate that the liver may be the first organ to store excessive amounts of fatty acids. Acting as a lipid buffer, the liver would have a detoxifying role in converting cytotoxic NEFA into TAG, a biologically inert molecule (Gibbons et al. 2004). Changes in liver lipid content can be caused by modification of lipid uptake which can then be directed towards lipid oxidation, lipid synthesis and storage, and/or 

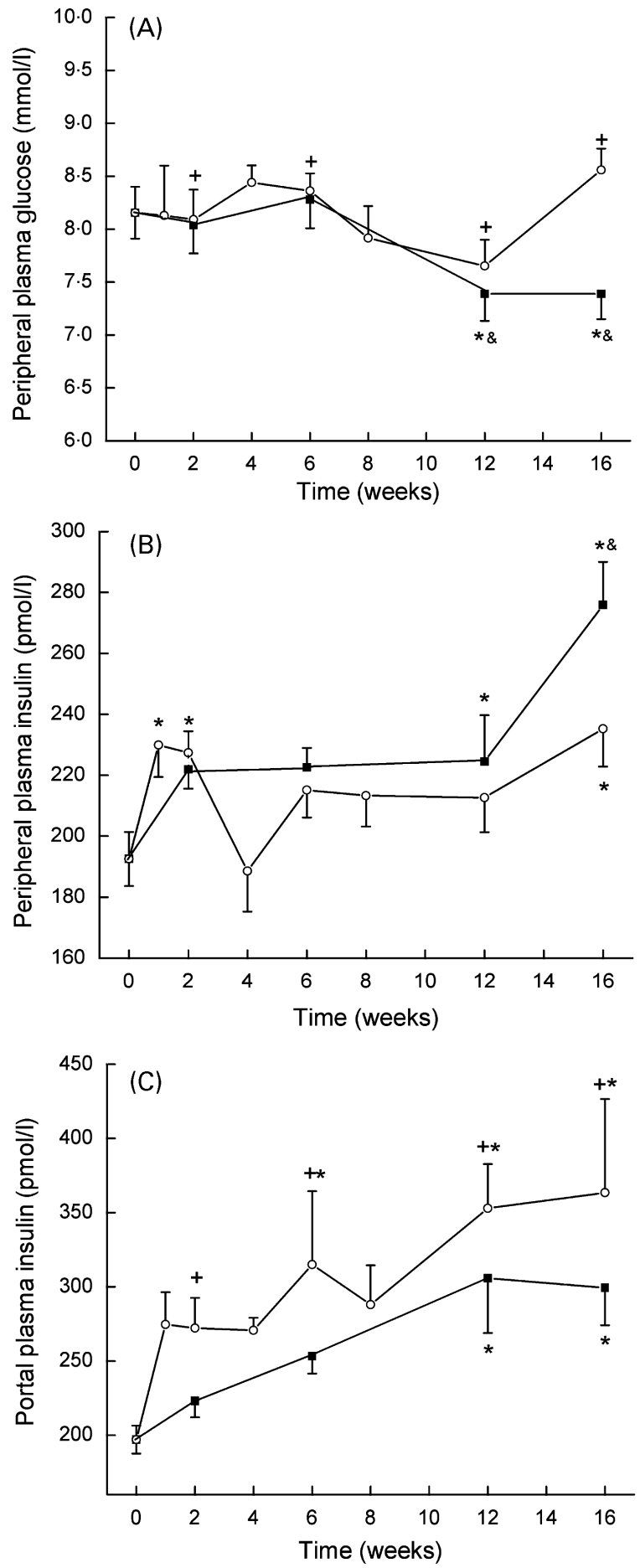

Fig. 4. Plasma glucose $(A)$ and plasma insulin concentrations measured in peripheral (B) and portal (C) blood in rats fed a standard ( $\square, n 9-10)$ or a high-fat $(\bigcirc, n 9-10)$ diet. For details of procedures, see p. 274. Values are means with their standard errors depicted by vertical bars. ${ }^{+}$Mean value significantly different from standard fed rats $(P<0.05)$. *Mean value significantly different from week $0(P<0.05)$. \&Mean value significantly different from week $6(P<0.05)$

changes in VLDL-TAG secretion by the organ. In the present study, it seems likely that the rapid and large accumulation of liver TAG observed during the first 2 weeks of high-fat feeding is mostly the result of an increase in NEFA uptake. Indeed, high-fat feeding resulted in a 3-fold increase in plasma NEFA levels after 2 weeks. The increased delivery of fatty acids to the liver should result in a higher uptake by the liver, because NEFA uptake is mostly done in a concentration-dependent manner (Burt et al. 1991). It is also important to mention that the higher energy intake (Fig. 1(A, B)) observed during the first weeks of high-fat feeding may have contributed to the rapid increase in plasma NEFA and liver fat content of $\mathrm{HF}$ rats. Furthermore, data from the present time-course study reveal that during the first 2 weeks of high-fat feeding, liver lipid partitioning seems directed more towards lipid synthesis and storage rather than lipid oxidation or VLDL-TAG secretion. On the other hand, the decrease and re-increase in liver lipid content between weeks 2 and 16 could be partly attributable to changes in VLDL-TAG secretion. Supporting this interpretation is the observation that, on the whole, the time course of plasma TAG, which can partly reflect VLDLTAG secretion, is opposite to the time course of liver TAG accumulation. It is becoming clear that liver TAG, which serve as precursors for secreted VLDL-TAG particles, do not come from newly synthesized TAG made from circulating NEFA but from a second pool of TAG located in the endoplasmic reticulum (Gibbons et al. 2004). This second pool of TAG, utilized for the assembly of VLDL-TAG particles, comes from the hydrolysis and subsequent re-esterification of cytosolic TAG, and thus requires a more complex enzymatic apparatus. It is tempting to postulate that upon an increased ingestion of fat, lipids are first preferentially partitioned towards the cytosolic pool, increasing liver TAG content, and that more time is required for the up-regulation of VLDL-TAG assembly and secretion.

The time course of energy intake could also be part of the explanation for the decrease in liver fat content seen between weeks 2 and 6 of high-fat feeding. After the first week, daily energy intake of HF rats slowly decreased and stabilized to the levels of SD rats. However, rats that were high-fat fed for 16 weeks showed significant hepatic steatosis while their mean daily energy intake calculated on the whole period of feeding was the same as observed in SD rats (Fig. 1(A)). Moreover, $\mathrm{HF}-\mathrm{OR}$ rats showed equivalent liver lipid content as HF-OP rats in spite of lower energy intake (Fig. 5(B, E)). It thus appears that energy intake can influence liver fat content but is not the sole contributor since isoenergetic high-fat feeding can still induce hepatic steatosis as seen in HF rats at 16 weeks.

An interesting observation of the present study is that the higher gain in intra-abdominal fat pads weight (Fig. 2), whether sampled from the mesenteric, retroperitoneal or urogenital area, in HF compared to SD rats was established as early as 2 weeks after the diet was initiated. The difference in intra-abdominal fat between the two dietary groups then remained constant during the subsequent 14 weeks of the experiment, $\mathrm{HF}$ rats gaining no more fat than SD rats after the first 2 weeks. The same pattern of response is also observed for plasma leptin concentration, which is well known to be highly correlated with the gain in adiposity (Bahceci et al. 1999). These observations suggest that the gain in adiposity is rapidly established when rats are submitted to high-fat feeding and, secondly, that animals seem to adapt to high-fat feeding so as to control the gain in adiposity. HF rats had a higher daily energy intake during the first few weeks of feeding which declined to the level of SD 

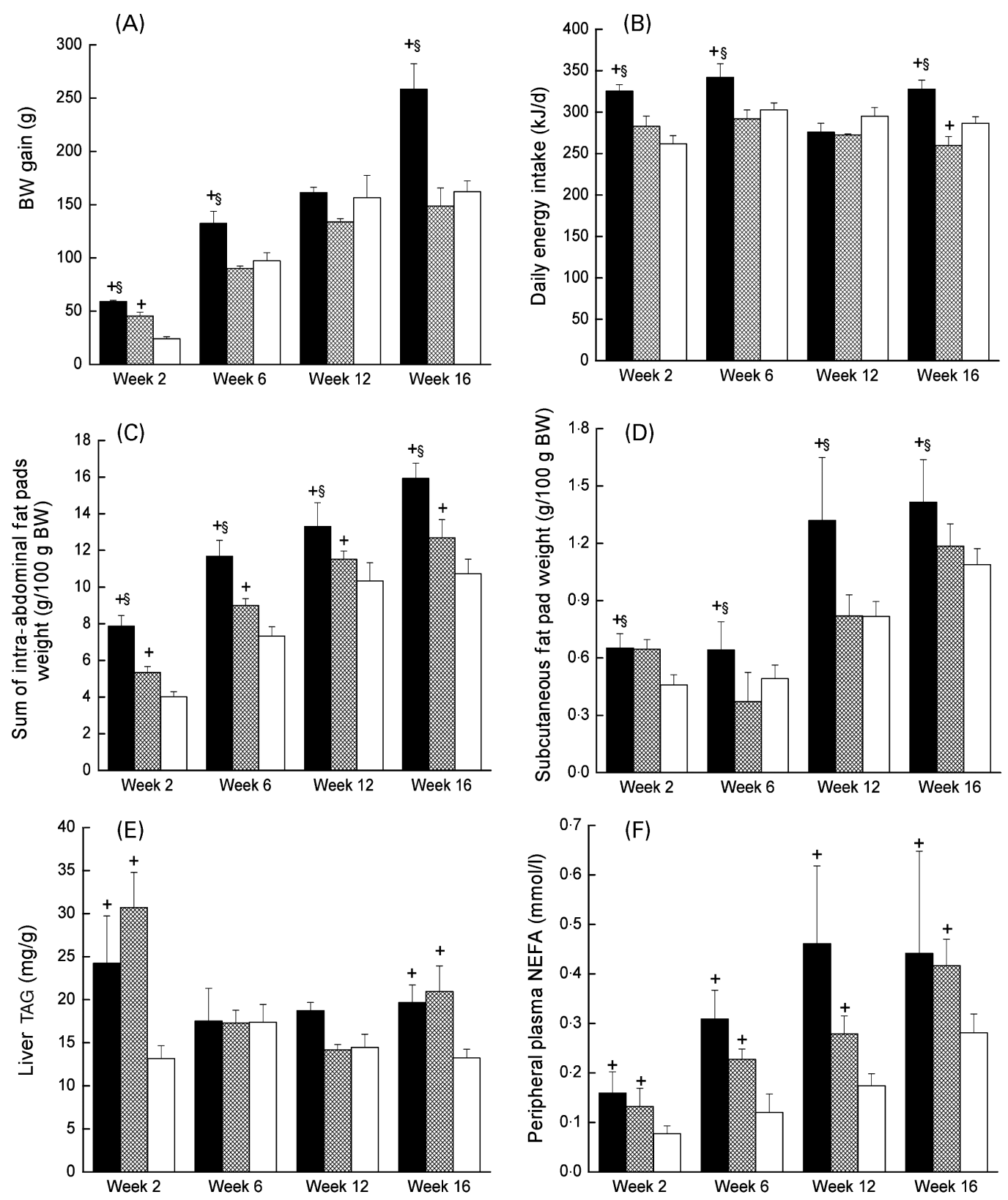

Fig. 5. Body weight gain (A), daily energy intake (B), sum of the relative weights of the three intra-abdominal fat pads (mesenteric, urogenital and retroperitoneal) (C), subcutaneous fat pad weight relative to $100 \mathrm{~g}$ body weight (BW) (D), liver triacylglycerol (TAG) concentration (E) and plasma NEFA concentration (F) of rats prone $(\square, n 5)$ or resistant $(\otimes, n 5)$ to high-fat diet-induced obesity and control rats on the standard diet $(\square, n 9)$. For details of procedures, see p. 274 . Values are means with their standard errors depicted by vertical bars. ${ }^{\S}$ Mean value significantly different from obesity-resistant rats $(P<0.05) .{ }^{+}$Mean value significantly different from standard fed rats $(P<0.05)$.

rats during the first 4 weeks (Fig. 1(A, B)). This initial hyperphagia could thus partly explain the time course of adipocyte fat accretion observed in the present study. Along with the rapid gain in adiposity, higher levels of portal insulin were established early ( 2 weeks) in HF compared to SD rats. Such an observation was not seen in peripheral blood. This may be taken as an indication that insulin secretion is rapidly increased by a high-fat diet and that consequently the liver is rapidly exposed to higher levels of insulin. These higher portal insulin levels in HF rats might have a role to play in the rapid development of hepatic steatotis.
In summary, results of the present study show that hepatic steatosis is a phenomenon that takes place rapidly when rats are submitted to high-fat feeding but that does not develop linearly over time. Moreover, obesisty-resistant rats do not seem to be protected against high-fat feeding-induced hepatic steatosis, suggesting a close relationship between dietary fat and liver lipid content, independent of body weight and fat accretion. From a clinical point of view, our results emphasize the importance of reducing dietary fat ingestion, even in the absence of a gain in body weight, to avoid the development of a state of hepatic steatosis. 


\section{Acknowledgements}

This work was supported by the Natural Sciences and Engineering Research Council of Canada (NSERC). Marie-Soleil Gauthier is supported by a Canada Graduate Scholarship from NSERC.

\section{References}

Axen KV, Dikeakos A \& Sclafani A (2003) High dietary fat promotes syndrome $\mathrm{X}$ in nonobese rats. J Nutr 133, 2244-2249.

Bahceci M, Tuzcu A, Akkus M, Yaldiz M \& Ozbay A (1999) The effect of high-fat diet on the development of obesity and serum leptin level in rats. Eat Weight Disord 4, 128-132.

Burt AD, MacSween RNM, Peters TJ \& Simpson KJ (1991) Nonalcoholic fatty liver: causes and complications. In Oxford Textbook of Clinical Hepatology, pp. 865-871 [N McIntyre, editor]. New York: Oxford University Press.

Chalkley SM, Hettiarachchi M, Chisholm DJ \& Kraegen EW (2002) Long-term high-fat feeding leads to severe insulin resistance but not diabetes in Wistar rats. Am J Physiol Endocrinol Metab 282, E1231-E1238.

Commerford SR, Pagliassotti MJ, Melby CL, Wei Y, Gayles EC \& Hill JO (2000) Fat oxidation, lipolysis, and free fatty acid cycling in obesity-prone and obesity-resistant rats. Am J Physiol Endocrinol Metab 279, E875-E885.

Day CP (2002) Pathogenesis of steatohepatitis. Best Pract Res Clin Gastroenterol 16, 663-678.

Frayn KN (2002) Adipose tissue as a buffer for daily lipid flux. Diabetologia 45, 1201-1210.

Frayn KN \& Maycock PF (1980) Skeletal muscle triacylglycerol in the rat: methods for sampling and measurement, and studies of biological variability. J Lipid Res 21, 139-144.

Gauthier MS, Couturier K, Charbonneau A \& Lavoie JM (2004) Effects of introducing physical training in the course of a 16week high-fat diet regimen on hepatic steatosis, adipose tissue fat accumulation, and plasma lipid profile. Int $J$ Obes Relat Metab Disord 28, 1064-1071.

Gauthier MS, Couturier K, Latour JG \& Lavoie JM (2003) Concurrent exercise prevents high-fat-diet-induced macrovesicular hepatic steatosis. J Appl Physiol 94, 2127-2134.

Ghibaudi L, Cook J, Farley C, van Heek M \& Hwa JJ (2002) Fat intake affects adiposity, comorbidity factors, and energy metabolism of Sprague-Dawley rats. Obes Res 10, 956-963.

Gibbons GF, Wiggins D, Brown AM \& Hebbachi AM (2004) Synthesis and function of hepatic very-low-density lipoprotein. Biochem Soc Trans 32, 59-64.

Hill JO, Lin D, Yakubu F \& Peters JC (1992) Development of dietary obesity in rats: influence of amount and composition of dietary fat. Int J Obes Relat Metab Disord 16, 321-333.

Ji H, Outterbridge LV \& Friedman MI (2005) Phenotype-based treatment of dietary obesity: differential effects of fenofibrate in obesity-prone and obesity-resistant rats. Metabolism 54, 421-429.

Kim JK, Gavrilova O, Chen Y, Reitman ML \& Shulman GI (2000) Mechanism of insulin resistance in A-ZIP/F-1 fatless mice. $J$ Biol Chem 275, 8456-8460.

Kraegen EW, Clark PW, Jenkins AB, Daley EA, Chisholm DJ \& Storlien LH (1991) Development of muscle insulin resistance after liver insulin resistance in high-fat-fed rats. Diabetes $\mathbf{4 0}$ $1397-1403$.

Krotkiewski M \& Bjorntorp P (1976) The effect of progesterone and of insulin administration on regional adipose tissue cellularity in the rat. Acta Physiol Scand 96, 122-127.

Lauterio TJ, Bond JP \& Ulman EA (1994) Development and characterization of a purified diet to identify obesity-susceptible and resistant rat populations. J Nutr 124, 2172-2178.

Levin BE, Dunn-Meynell AA, Balkan B \& Keesey RE (1997) Selective breeding for diet-induced obesity and resistance in SpragueDawley rats. Am J Physiol 273, R725-R730.

Lo S, Russell JC \& Taylor AW (1970) Determination of glycogen in small tissue samples. J Appl Physiol 28, 234-236.

Marceau P, Biron S, Hould FS, Marceau S, Simard S, Thung SN \& Kral JG (1999) Liver pathology and the metabolic syndrome X in severe obesity. J Clin Endocrinol Metab 84, 1513-1517.

Pagliassotti MJ, Knobel SM, Shahrokhi KA, Manzo AM \& Hill JO (1994) Time course of adaptation to a high-fat diet in obesityresistant and obesity-prone rats. Am J Physiol Regul Integr Comp Physiol 267, R659-R664.

Pagliassotti MJ, Pan D, Prach P, Koppenhafer T, Storlien L \& Hill JO (1995) Tissue oxidative capacity, fuel stores and skeletal muscle fatty acid composition in obesity-prone and obesity-resistant rats. Obes Res 3, 459-464.

Pagliassotti MJ, Shahrokhi KA \& Hill JO (1993) Skeletal muscle glucose metabolism in obesity-prone and obesityresistant rats. Am J Physiol Regul Integr Comp Physiol 264, R1224-R1228.

Reue K, Xu P, Wang XP \& Slavin BG (2000) Adipose tissue deficiency, glucose intolerance, and increased atherosclerosis result from mutation in the mouse fatty liver dystrophy (fld) gene. J Lipid Res 41, 1067-1076.

Saltiel AR \& Kahn CR (2001) Insulin signalling and the regulation of glucose and lipid metabolism. Nature 414, 799-806.

Samuel VT, Liu Z-X, Qu X, Elder BD, Bilz S, Befroy D, Romanelli AJ \& Shulman GI (2004) Mechanism of hepatic insulin resistance in non-alcoholic fatty liver disease. $J$ Biol Chem 279, 32345-32353.

Satia-Abouta J, Patterson RE, Schiller RN \& Kristal AR (2002) Energy from fat is associated with obesity in U.S. men: results from the Prostate Cancer Prevention Trial. Prev Med 34, $493-501$.

Seppala-Lindroos A, Vehkavaara S, Hakkinen AM, Goto T, Westerbacka J, Sovijarvi A, Halavaara J \& Yki-Jarvinen H (2002) Fat accumulation in the liver is associated with defects in insulin suppression of glucose production and serum free fatty acids independent of obesity in normal men. J Clin Endocrinol Metab 87, 3023-3028.

Sniderman AD, Cianflone K, Arner P, Summers LKM \& Frayn KN (1998) The adipocyte, fatty acid trapping, and atherogenesis. Arterioscler Thromb Vasc Biol 18, 147-151.

Wang J, Alexander JT, Zheng P, Yu HJ, Dourmashkin J \& Leibowitz SF (1998) Behavioral and endocrine traits of obesity-prone and obesity-resistant rats on macronutrient diets. Am J Physiol Endocrinol Metab 274, E1057-E1066.

Westerbacka J, Lammi K, Hakkinen AM, Rissanen A, Salminen I, Aro A \& Yki-Jarvinen H (2005) Dietary fat content modifies liver fat in overweight non-diabetic subjects. $J$ Clin Endocrinol Metab 90(5), 2804-2809. 